\title{
The porous medium equation as the relaxation limit of the isentropic Euler equations
}

\author{
Hongli Wang ${ }^{\mathrm{a}, *}$, Huimin Liu ${ }^{\mathrm{b}}$ \\ a College of Mathematics and Information Science, \\ North China University of Water Resources and Electric Power, Zhengzhou 450045, Henan Province, \\ China \\ b General Subjects Department, Zhengzhou Chenggong University of Finance and Economics, \\ Zhengzhou 451200, Henan Province, China
}

*Corresponding author, e-mail: wanghongli@ncwu.edu.cn

Received 27 Aug 2014

Accepted 25 Apr 2015

\begin{abstract}
This work is concerned with the relaxation limits of compressible and isentropic Euler equations. For well prepared initial data, the convergence of solution of the compressible Euler equations to the solution of the porous medium equation is justified by an analysis of asymptotic expansions up to any order and a uniform energy estimate.
\end{abstract}

KEYWORDS: small parameter limit, energy estimate, asymptotic expansions

MSC2010: 35L45 76N15 35L65

\section{INTRODUCTION}

Let $n(x, t)$ and $u(x, t)$ be the fluid density function and the fluid velocity. They are functions of a three-dimensional position vector $x \in \mathbb{R}^{3}$ and of the time $t>0$. With a suitable scaling change, the compressible Euler equations with relaxation are

$$
\begin{gathered}
\partial_{t} n+\frac{1}{\tau} \operatorname{div}(n u)=0, \\
\partial_{t}(n u)+\frac{1}{\tau} \operatorname{div}(n u \otimes u)+\frac{1}{\tau} \nabla P=-\frac{n u}{\tau^{2}}, \\
\left.(n, u)\right|_{t=0}=\left(n_{0}^{\tau}, u_{0}^{\tau}\right) .
\end{gathered}
$$

Here, $\tau$ is the momentum relaxation time for some physical flow and we assume that $0<\tau \ll 1$; the pressure $P(n)$ is given as the $\gamma$-law: $P(n)=a^{2} n^{\gamma}$, where the case $\gamma>1$ corresponds to isentropic flow and $\gamma=1$ corresponds to the isothermal flow; and $a^{2}$ is a constant. For smooth solutions with $n>0$, (1b) is equivalent to

$$
\partial_{t} u+\frac{1}{\tau}(u \cdot \nabla) u+\frac{1}{\tau} \nabla h(n)=-\frac{u}{\tau^{2}},
$$

where the enthalpy function

$$
h(n)=\int_{1}^{n} \frac{P^{\prime}(s)}{s} \mathrm{~d} s .
$$

Since $P$ is sufficiently smooth and strictly increasing on $(0,+\infty)$, so is $h$.
In (1), $\tau$ is very small compared to the other quantities. When the relaxation time $\tau$ tends to 0 , we will show that the density converges towards the solution to the porous medium equation. The problem of the above limit has been justified by many authors. The convergence of the solutions to (1) towards those of a heat equation for arbitrary large initial data has been studied in $B V(\mathbb{R})$ space $^{1}$. In Ref. 2 , the derivation of the porous media equation as the limit of the isentropic Euler equations in one space dimension was shown. In Ref. 3 the uniform smooth solutions to (1) were constructed in the multidimensional case and this relaxation-time limit was proved in some Sobolev space. As the relaxation time tends to zero, the density of certain scaled isentropic Euler equations with relaxation strongly converges towards the smooth solution to the porous medium equationRef. 4 . We also refer to Ref. 5 where the convergence for the smooth solution of the compressible Euler-Maxwell equations to the smooth solution of the drift-diffusion equations is proved by means of Maxwell iteration, as the relaxation time tends to zero.

In this paper, we are still interested in the zerorelaxation limit $\tau \rightarrow 0$. Here we state the main difference between the present paper and Ref. 4 . The convergence of the asymptotic expansion was proved in Ref. 4 only up to first order and our asymptotic expansions are justified up to any order. 
We note that if we replace $u$ by $\tau u$, then (1) becomes

$$
\begin{gathered}
\partial_{t} n+\operatorname{div}(n u)=0, \\
\partial_{t} u+(u \cdot \nabla) u+\frac{1}{\tau^{2}} \nabla h(n)=-\frac{u}{\tau^{2}},
\end{gathered}
$$

in which the only small parameter is $\tau^{2}$. Thus we propose an asymptotic expansion to (1) of the form which has only even powers of the relaxation time:

$$
\left(n_{m}^{\tau}, u_{m}^{\tau}\right)=\sum_{j=0}^{m} \tau^{2 j}\left(n^{j}, \tau u^{j}\right),
$$

where the leading profiles $\left(n^{0}, u^{0}\right)$ satisfy a porous medium equation as shown in Ref. 4. With (2), for $m \geqslant 0$ we prove the convergence of the solution $\left(n^{\tau}, u^{\tau}\right)$ of (1) to $\left(n_{m}^{\tau}, u_{m}^{\tau}\right)$ with a higher order $O\left(\tau^{2(m+1)}\right)$ when the initial data are well-prepared and the initial errors have the same order.

Finally, we mention that the asymptotic expansions used in this paper resemble the ones that arise in the study of the fluid limits from the Boltzmann equations. We refer to Refs. 6-8 and the references therein for this topic.

The remainder of this paper is organized as follows. We derive asymptotic expansions of solutions and state the convergence result to problem (1) in the case of well-prepared initial data. The justification of the asymptotic expansions is then given. For this purpose, we prove a more general convergence theorem which implies the convergence of the expansions.

\section{ASYMPTOTIC ANALYSIS}

In this section we consider the zero-relaxation limit $\tau \rightarrow 0$ in a problem with well-prepared initial data. First, we recall the local existence of smooth solutions for symmetrizable hyperbolic equations.

Proposition 1 (Local existence of smooth solutions ${ }^{9,10}$ ) Let $s \geqslant 3$ be an integer and $\left(n_{0}^{\tau}, u_{0}^{\tau}\right) \in$ $H^{s}\left(\mathbb{R}^{3}\right)$ with $n_{0}^{\tau} \geqslant \bar{n}$ for some given constant $\bar{n}>0$, independent of $\tau$. Then there exist $T_{1}^{\tau}>0$ and a unique smooth solution $\left(n^{\tau}, u^{\tau}\right)$ to the Cauchy problem (1) defined in the time interval $\left[0, T_{1}^{\tau}\right]$ with

$$
\left(n^{\tau}, u^{\tau}\right) \in C^{k}\left(\left[0, T_{1}^{\tau}\right] ; H^{s+1-k}\left(\mathbb{R}^{3}\right)\right), \quad k=1,2 .
$$

Let $\left(n^{\tau}, u^{\tau}\right)$ be the classical solutions to (1). Now we study its formal asymptotic expansions with respect to $\tau \in\left(0, \tau_{0}\right]$ for a certain small $\tau_{0}$. Based on the discussion on the asymptotic expansion, we make the following ansatz for both the approximate solution and its initial data:

$$
\begin{aligned}
& \left(n^{\tau}, u^{\tau}\right)(x, 0)=\sum_{j \geqslant 0} \tau^{2 j}\left(n_{j}, \tau u_{j}\right)(x), \\
& \left(n^{\tau}, u^{\tau}\right)(x, t)=\sum_{j \geqslant 0} \tau^{2 j}\left(n^{j}, \tau u^{j}\right),
\end{aligned}
$$

where the sequences $\left(n_{j}, u_{j}\right)_{j \geqslant 0}$ are given sufficiently smooth data with $n_{0} \geqslant$ constant $>0$ in $\mathbb{R}^{3}$. The validity of (3) is discussed in the next section.

Now let us determine the profiles $\left(n^{j}, u^{j}\right)$ for all $j \geqslant 0$. Putting (3b) into (1) and identifying the coefficients in powers of $\tau$, we obtain a series of equations verified by the profiles $\left(n^{j}, u^{j}\right)_{j \geqslant 0}$.

The leading profiles $\left(n^{0}, u^{0}\right)$ satisfy the following equations:

$$
\begin{gathered}
\partial_{t} n^{0}+\operatorname{div}\left(n^{0} u^{0}\right)=0 \\
n^{0} u^{0}=-\nabla\left(P\left(n^{0}\right)\right) .
\end{gathered}
$$

Then $n^{0}$ solves a parabolic equation

$$
\begin{gathered}
\partial_{t} n^{0}-\Delta\left(P\left(n^{0}\right)\right)=0, \\
n^{0}(x, 0)=n_{0}, x \in \mathbb{R}^{3} .
\end{gathered}
$$

The existence of smooth solutions to problem (5) can be easily established, at least locally in time.

From (5b) and (4b) we get the first order compatibility conditions:

$$
u_{0}=-\frac{1}{n_{0}} \nabla\left(P\left(n_{0}\right)\right)=-\nabla h\left(n_{0}\right) .
$$

In general, for any $j \geqslant 1$, the profiles $\left(n^{j}, u^{j}\right)$ can be obtained by induction in $j$. Now we assume that $\left(n^{k}, u^{k}\right)_{0 \leqslant k \leqslant j-1}$ are smooth and already determined in previous steps. Then $\left(n^{j}, u^{j}\right)$ satisfy the following linearized equations:

$$
\left.\begin{array}{c}
\partial_{t} n^{j}+\operatorname{div}\left(n^{0} u^{j}+n^{j} u^{0}\right)=-\sum_{k=1}^{j-1} \operatorname{div}\left(n^{k} u^{j-k}\right), \\
\partial_{t} u^{j-1}+\sum_{k=0}^{j-1}\left(u^{k} \cdot \nabla\right) u^{j-k} \\
+\nabla\left(h^{\prime}\left(n^{0}\right) n^{j}+h^{j-1}\left(\left(n^{k}\right)_{k \leqslant j-1}\right)\right)=-u^{j},
\end{array}\right\}
$$

where $h^{0}=0$ and $h^{j-1}\left(\left(n^{k}\right)_{k \leqslant j-1}\right)$ is a smooth function with respect to its arguments $\left(n^{k}\right)_{k \leqslant j-1}$ and can be obtained from the following relation for $j \geqslant 2$ :

$$
h\left(\sum_{j \geqslant 0} \tau^{2 j} n^{j}\right)=\sum_{j \geqslant 0} c_{j} \tau^{2 j},
$$


with

$$
\begin{gathered}
c_{0}=h\left(n^{0}\right), c_{1}=h^{\prime}\left(n^{0}\right) n^{1}, \\
c_{j}=h^{\prime}\left(n^{0}\right) n^{j}+h^{j-1}\left(\left(n^{k}\right)_{k \leqslant j-1}\right), \forall j \geqslant 2 .
\end{gathered}
$$

Hence $n^{j}$ solves a linearized system of porous medium equations for $0 \leqslant k \leqslant j-1$ :

$$
\begin{aligned}
& \partial_{t} n^{j}-\operatorname{div}\left(n^{0} \nabla\left(h^{\prime}\left(n^{0}\right) n^{j}\right)\right)+\operatorname{div}\left(n^{j} u^{0}\right) \\
& \quad=g^{j-1}\left(V^{k}, \partial_{t} V^{k}, \partial_{x} V^{k}, \partial_{t} \partial_{x} V^{k}, \partial_{x}^{2} V^{k}\right),
\end{aligned}
$$

with the initial condition

$$
n^{j}(x, 0)=n_{j}(x), x \in \mathbb{R}^{3},
$$

where $g^{j-1}$ is a given smooth function and $V^{k}=$ $\left(n^{k}, u^{k}\right)$. Problem (8) with (9) is linear. It admits a unique global smooth solution. Then $u^{j}$ is given by (7). Thus we get the high-order compatibility conditions for $j \geqslant 1$

$$
\begin{aligned}
u_{j}=-\partial_{t} u_{j-1}-\sum_{k=0}^{j-1}\left(u_{k} \cdot \nabla\right) u_{j-k} \\
+\nabla\left(h^{\prime}\left(n_{0}\right) n_{j}+h^{j-1}\left(\left(n_{k}\right)_{k \leqslant j-1}\right)\right) .
\end{aligned}
$$

We conclude the above discussion with the following result.

Proposition 2 Let $s \geqslant 3$ be an integer. Assume $\left(n_{j}, u_{j}\right) \in H^{s+1}\left(\mathbb{R}^{3}\right)$ for $j \geqslant 0$, with $n_{0} \geqslant$ constant $>0$ in $\mathbb{R}^{3}$, and the compatibility conditions (6) for $j=0$ and (10) for $j \geqslant 1$ are satisfied. Then there exists a unique asymptotic expansion up to any order of the form (3b), i.e., there exist $T_{1}>0$ and a unique smooth solution $\left(n^{j}, u^{j}\right)_{j \geqslant 0}$ in the time interval $\left[0, T_{1}\right]$ of (5) for $j=0$ and (8) with (9) for $j \geqslant 1$. Moreover, $n^{0} \geqslant$ cons $\tan t>0$ in $\mathbb{R}^{3} \times\left[0, T_{1}\right]$ and

$$
\left(n^{j}, u^{j}\right) \in C^{k}\left(\left[0, T_{1}\right] ; H^{s+1-k}\left(\mathbb{R}^{3}\right)\right), k=1,2, j \geqslant 0 .
$$

In particular, the formal zero-relaxation limit $\tau \rightarrow$ 0 of the isentropic Euler system (1) is the porous medium equation (5a) and (8).

For any fixed integers $m \geqslant 0$ we denote by

$$
\left(n_{m}^{\tau}, u_{m}^{\tau}\right)=\sum_{j=0}^{m} \tau^{2 j}\left(n^{j}, \tau u^{j}\right),
$$

an approximate solution of order $m$, where $\left(n^{j}, u^{j}\right)_{0 \leqslant j \leqslant m}$ were constructed in the previous subsection. We define the remainders $R_{n, m}^{\tau}$ and $R_{u, m}^{\tau}$ by

$$
\left.\begin{array}{c}
\partial_{t} n_{m}^{\tau}+\frac{1}{\tau} \operatorname{div}\left(n_{m}^{\tau} u_{m}^{\tau}\right)=R_{n, m}^{\tau}, \\
\partial_{t} u_{m}^{\tau}+\frac{1}{\tau}\left(u_{m}^{\tau} \cdot \nabla\right) u_{m}^{\tau}+\frac{1}{\tau} \nabla h\left(n_{m}^{\tau}\right) \\
=-\frac{u_{m}^{\tau}}{\tau^{2}}+R_{u, m}^{\tau} .
\end{array}\right\}
$$

It is clear that the convergence rate depends strongly on the order of the remainders with respect to $\tau$. It is easy to see that

$$
R_{n, m}^{\tau}=O\left(\tau^{2 m+1}\right), R_{u, m}^{\tau}=O\left(\tau^{2 m+1}\right) .
$$

In (13), there is a loss of one order for the remainder $R_{u, m}^{\tau}$ and this loss will be recovered in the error estimate of convergence due to the dissipation term for $u$.

Proposition 3 Let the assumptions of Proposition 2 hold. For all integers $m \geqslant 0$ and $s \geqslant 3$ the remainders $R_{n, m}^{\tau}, R_{u, m}^{\tau}$ and

$$
\begin{aligned}
& \sup _{0 \leqslant t \leqslant T_{1}}\left\|R_{n, m}^{\tau}\right\|_{s} \leqslant C_{m} \tau^{2(m+1)}, \\
& \sup _{0 \leqslant t \leqslant T_{1}}\left\|R_{u, m}^{\tau}\right\|_{s} \leqslant C_{m} \tau^{2 m+1},
\end{aligned}
$$

where $C_{m}>0$ is a constant independent of $\tau$.

The convergence result of this section is given in the following theorem. The proof is given in the next section.

Theorem 1 Let $m \geqslant 0$ and $s \geqslant 3$ be any fixed integers. Let the assumptions of Proposition 2 hold. We suppose

$$
\left\|\left(n_{0}^{\tau}, u_{0}^{\tau}\right)-\sum_{j=0}^{m} \tau^{2 j}\left(n_{j}, \tau u_{j}\right)\right\|_{s} \leqslant C_{1} \tau^{2(m+1)},
$$

where $C_{1}>0$ is a constant independent of $\tau$. Then there exists a constant $C_{2}>0$, independent of $\tau$, such that as $\tau \rightarrow 0$ we have $T_{1}^{\tau} \geqslant T_{1}$ and the solution $\left(n^{\tau}, u^{\tau}\right)$ to the Cauchy problem (1) satisfies

$$
\left\|\left(n^{\tau}, u^{\tau}\right)-\left(n_{m}^{\tau}, u_{m}^{\tau}\right)\right\|_{s} \leqslant C_{2} \tau^{2(m+1)}
$$

for all $t \in\left[0, T_{1}\right]$.

Moreover, we have that

$$
\left\|u^{\tau}-u_{m}^{\tau}\right\|_{L^{2}\left(\left[0, T_{1}\right], H^{s}\left(\mathbb{R}^{3}\right)\right)} \leqslant C_{2} \tau^{2 m+3} .
$$

\section{MAIN RESULT}

In this section, we give a more general convergence result which implies Theorem 1 .

Let $\left(n^{\tau}, u^{\tau}\right)$ be the exact solution to (1) with initial data $\left(n_{0}^{\tau}, u_{0}^{\tau}\right)$ and let $\left(n_{\tau}, u_{\tau}\right)$ be an approximate periodic solution defined on $\left[0, T_{1}\right]$ with

$$
\left(n_{\tau}, u_{\tau}\right) \in C^{k}\left(\left[0, T_{1}\right] ; H^{s+1-k}\left(\mathbb{R}^{3}\right)\right), \quad k=1,2 .
$$

We define the remainders of the approximate solution by

$$
\begin{aligned}
R_{n}^{\tau} & =\partial_{t} n_{\tau}+\frac{1}{\tau} \operatorname{div}\left(n_{\tau} u_{\tau}\right), \\
R_{u}^{\tau} & =\partial_{t} u_{\tau}+\frac{1}{\tau}(u \cdot \nabla) u_{\tau}+\frac{1}{\tau} \nabla h\left(n_{\tau}\right)+\frac{u_{\tau}}{\tau^{2}} .
\end{aligned}
$$


We suppose that

$$
\begin{gathered}
\sup _{0 \leqslant t \leqslant T_{1}}\left\|n_{\tau}\right\|_{s} \leqslant C_{1}, \sup _{0 \leqslant t \leqslant T_{1}}\left\|u_{\tau}\right\|_{s} \leqslant C_{1} \tau, \\
\left\|\left(n_{0}^{\tau}, u_{0}^{\tau}\right)-\left(n_{\tau 0}(x, 0), u_{\tau 0}\right)\right\|_{s} \leqslant C_{1} \tau^{\lambda+1}, \\
\sup _{0 \leqslant t \leqslant T_{1}}\left\|R_{n}^{\tau}\right\|_{s} \leqslant C_{1} \tau^{\lambda+1}, \sup _{0 \leqslant t \leqslant T_{1}}\left\|R_{u}^{\tau}\right\|_{s} \leqslant C_{1} \tau^{\lambda},
\end{gathered}
$$

where $\lambda \geqslant 0$ and $C_{1}>0$ are constants independent of $\tau$.

Theorem 2 Let $s \geqslant 3$ be an integer and $\lambda \geqslant 0$. Under the above assumptions, there exists a constant $C_{2}>0$, independent of $\tau$, such that as $\tau \rightarrow 0$ we have $T_{1}^{\tau} \geqslant T_{1}$ and the solution $\left(n^{\tau}, u^{\tau}\right)$ of (1) satisfies

$$
\left\|\left(n^{\tau}, u^{\tau}\right)(t)-\left(n_{\tau}, u_{\tau}\right)(t)\right\|_{s} \leqslant C_{2} \tau^{\lambda+1}
$$

for all $t \in\left[0, T_{1}\right]$. Moreover, we have that

$$
\left\|u^{\tau}-u_{\tau}\right\|_{L^{2}\left(\left[0, T_{1}\right] ; H^{s}\left(\mathbb{R}^{3}\right)\right)} \leqslant C_{2} \tau^{\lambda+2} .
$$

\section{PROOF OF THE MAIN RESULT}

To start with, we recall the Moser-type calculus inequalities in Sobolev spaces for later use in this section.

Lemma 1 (Moser-type calculus inequalities ${ }^{10,11}$ ) Let $s \geqslant 1$ be an integer. Suppose $u \in H^{s}\left(\mathbb{R}^{3}\right)$, $\nabla u \in L^{\infty}\left(\mathbb{R}^{3}\right)$, and $v \in H^{s-1}\left(\mathbb{R}^{3}\right) \cap L^{\infty}\left(\mathbb{R}^{3}\right)$. Then for all multi-indexes $|\alpha| \leqslant s$, we have $\left(\partial_{x}^{\alpha}(u v)-u \partial_{x}^{\alpha} v\right) \in L^{2}\left(\mathbb{R}^{3}\right)$ and

$$
\begin{array}{r}
\left\|\partial_{x}^{\alpha}(u v)-u \partial_{x}^{\alpha} v\right\| \leqslant C_{s}\left(\|\nabla u\|_{0, \infty}\left\|D^{|\alpha|-1} v\right\|\right. \\
\left.+\left\|D^{|\alpha|} u\right\|\|v\|_{0, \infty}\right)
\end{array}
$$

where

$$
\left\|D^{h} u\right\|=\sum_{|\alpha|=h}\left\|\partial_{x}^{\alpha} u\right\|, \forall h \in \mathbb{N}
$$

and

$$
\partial_{x}^{\alpha}=\frac{\partial^{|\alpha|}}{\partial x_{1}^{\alpha_{1}} \cdot \partial x_{2}^{\alpha_{2}} \cdot \partial x_{3}^{\alpha_{3}}} \text { with }|\alpha|=\sum_{k=1}^{3}\left|\alpha_{k}\right| .
$$

Moreover, the embedding $H^{s-1}\left(\mathbb{R}^{3}\right) \hookrightarrow L^{\infty}\left(\mathbb{R}^{3}\right)$ for $s \geqslant 3$ is continuous. Hence we have

$$
\begin{gathered}
\|u v\|_{s-1} \leqslant C_{s}\|u\|_{s-1}\|v\|_{s-1}, \\
\left\|\partial_{x}^{\alpha}(u v)-u \partial_{x}^{\alpha} v\right\| \leqslant C_{s}\|u\|_{s}\|v\|_{s-1} .
\end{gathered}
$$

By Proposition 1, the exact solution $\left(n^{\tau}, u^{\tau}\right)$ is defined in a time interval $\left[0, T_{1}^{\tau}\right]$ with $T_{1}^{\tau}>0$. Since $n^{\tau} \in C\left(\left[0, T_{1}^{\tau}\right] ; H^{s}\left(\mathbb{R}^{3}\right)\right)$ and the embedding from $H^{s}\left(\mathbb{R}^{3}\right)$ to $C\left(\mathbb{R}^{3}\right)$ is continuous, we have $n^{\tau} \in$
$C\left(\mathbb{R}^{3} \times\left[0, T_{1}^{\tau}\right]\right)$. From (18) and (19) and assumption $n_{0}^{\tau} \geqslant \bar{n}>0$, we deduce that there exist $T_{2}^{\tau} \in\left(0, T_{1}^{\tau}\right]$ and a constant $C_{0}>0$, independent of $\tau$, such that

$$
\frac{\bar{n}}{2} \leqslant n^{\tau}(x, t) \leqslant C_{0}, \forall(x, t) \in \mathbb{R}^{3} \times\left(0, T_{1}^{\tau}\right] .
$$

Similarly, the function $t \mapsto\left\|\left(n^{\tau}, u^{\tau}\right)(t)\right\|_{s}$ is continuous in $C\left(\left[0, T_{2}^{\tau}\right]\right)$. From (18), we know that the sequence $\left(\left\|\left(n^{\tau}(x, 0), u^{\tau}(x, 0)\right)\right\|_{s}\right)_{\tau>0}$ is bounded. Then there exist $T_{3}^{\tau}\left(0, T_{2}^{\tau}\right]$ and a constant, still denoted by $C_{0}>0$, such that

$$
\left\|\left(n^{\tau}(\cdot, t), u^{\tau}(\cdot, t)\right)\right\|_{s} \leqslant C_{0}, \forall t \in\left(0, T_{3}^{\tau}\right] .
$$

Then we define $T^{\tau}=\min \left(T_{1}, T_{3}^{\tau}\right)>0$ so that the exact solution and the approximate solution are both defined in the time interval $\left[0, T^{\tau}\right]$. In this time interval, we set

$$
\left(N^{\tau}, U^{\tau}\right)=\left(n^{\tau}-n_{\tau}, u^{\tau}-u_{\tau}\right)
$$

From (1) and (17) it follows that the error $\left(N^{\tau}, U^{\tau}\right)$ satisfies the following problem

$$
\left.\begin{array}{r}
\partial_{t} N^{\tau}+\frac{1}{\tau} u^{\tau} \cdot \nabla N^{\tau}+\frac{1}{\tau} n^{\tau} \operatorname{div} U^{\tau} \\
=-\frac{1}{\tau}\left(U^{\tau} \cdot \nabla n_{\tau}+N^{\tau} \operatorname{div} u_{\tau}\right)-R_{n}^{\tau}, \\
\partial_{t} U^{\tau}+\frac{1}{\tau}\left(u^{\tau} \cdot \nabla\right) U^{\tau}+\frac{1}{\tau} h^{\prime}\left(n^{\tau}\right) \nabla N^{\tau} \\
=-\frac{1}{\tau}\left[\left(h^{\prime}\left(n^{\tau}\right)-h^{\prime}\left(n_{\tau}\right)\right) \nabla n_{\tau}\right] \\
-\frac{1}{\tau}\left(U^{\tau} \cdot \nabla\right) u_{\tau}-\frac{U^{\tau}}{\tau^{2}}-R_{u}^{\tau}, \\
\left.\left(N^{\tau}, U^{\tau}\right)\right|_{t=0}=\left(n_{0}^{\tau}-n_{\tau 0}, u_{0}^{\tau}-u_{\tau 0}\right) .
\end{array}\right\}
$$

Set

$$
\begin{aligned}
& W^{\tau}=\left(\begin{array}{l}
N^{\tau} \\
U^{\tau}
\end{array}\right), \\
& A_{i}^{\tau}=\left(\begin{array}{cc}
u_{i}^{\tau} & n^{\tau} \mathrm{e}_{i}^{t} \\
h^{\prime}\left(n^{\tau}\right) e_{i} & u_{i}^{\tau} I_{3}
\end{array}\right), i=1,2,3, \\
& H_{1}=\left(\begin{array}{c}
-\left(U^{\tau} \cdot \nabla n_{\tau}+N^{\tau} \operatorname{divu}_{\tau}\right) \\
-\left(U^{\tau} \cdot \nabla\right) u_{\tau}-\left(h^{\prime}\left(n^{\tau}\right)-h^{\prime}\left(n_{\tau}\right)\right) \nabla n_{\tau}
\end{array}\right), \\
& H_{2}=\left(\begin{array}{c}
0 \\
-U^{\tau}
\end{array}\right), R^{\tau}=\left(\begin{array}{c}
R_{n}^{\tau} \\
R_{u}^{\tau}
\end{array}\right) \text {, }
\end{aligned}
$$

where $\left(e_{1}, e_{2}, e_{3}\right)$ is the canonical base of $\mathbb{R}^{3}, I_{3}$ is the $3 \times 3$ identity matrix, $y_{i}$ denotes the $i$ th component of $y \in \mathbb{R}^{3}$. Thus (25) for the unknown $W^{\tau}$ can be rewritten as

$$
\partial_{t} W^{\tau}+\frac{1}{\tau} \sum_{i=1}^{3} A_{i}^{\tau} \partial_{x_{i}} W^{\tau}=\frac{1}{\tau} H_{1}+\frac{1}{\tau^{2}} H_{2}+R^{\tau} .
$$


It is symmetrizable hyperbolic with

$$
A_{0}^{\tau}\left(n^{\tau}\right)=\left(\begin{array}{cc}
\left(n^{\tau}\right)^{-1} & 0 \\
0 & \left(h^{\prime}\left(n^{\tau}\right)\right)^{-1}
\end{array}\right)
$$

as its symmetrizer. Then

$$
\widetilde{A}_{i}^{\tau}=A_{0}^{\tau}\left(n^{\tau}\right) A_{i}^{\tau}=u_{i}^{\tau} A_{0}^{\tau}\left(n^{\tau}\right)+D_{i}
$$

is symmetric for all $1 \leqslant i \leqslant 3$, where each $D_{i}$ is a constant matrix

$$
D_{i}=\left(\begin{array}{cc}
0 & \mathrm{e}_{i}^{t} \\
e_{i} & 0
\end{array}\right), i=1,2,3 .
$$

The existence and uniqueness of smooth solutions to (1) are equivalent to that of (25). Thus in order to prove Theorem 2, it suffices to establish uniform estimates of $W^{\tau}$ with respect to $\tau$. In what follows, we denote by $C>0$ various constants independent of $\tau$ and for $\alpha \in \mathbb{N}^{3}, W_{\alpha}^{\tau}=\partial_{x}^{\alpha} W^{\tau}$, etc.

Lemma 2 Under the conditions of Theorem 2, we have

$$
\begin{aligned}
& \left\|W^{\tau}(t)\right\|_{s}^{2}+\frac{1}{\tau^{2}} \int_{0}^{t}\left\|U^{\tau}(\xi)\right\|_{s}^{2} \mathrm{~d} \xi \\
& \quad \leqslant C \int_{0}^{t}\left(\left\|W^{\tau}(\xi)\right\|_{s}^{2}+\left\|W^{\tau}(\xi)\right\|_{s}^{4}\right) \mathrm{d} \xi+C \tau^{2(\lambda+1)} .
\end{aligned}
$$

Proof: Taking $\partial_{x}^{\alpha}$ of the above equations for a multiindex $\alpha$ satisfying $|\alpha| \leqslant s$, one gets

$$
\begin{aligned}
\partial_{t} W_{\alpha}^{\tau} & +\frac{1}{\tau} \sum_{i=1}^{3} A_{i}^{\tau} \partial_{x_{i}} W_{\alpha}^{\tau} \\
= & \frac{1}{\tau} \partial_{x}^{\alpha} H_{1}+\frac{1}{\tau^{2}} \partial_{x}^{\alpha} H_{2}+\partial_{x}^{\alpha} R^{\tau} \\
& +\frac{1}{\tau} \sum_{i=1}^{3}\left[A_{i}^{\tau} \partial_{x_{i}} W_{\alpha}^{\tau}-\partial_{x}^{\alpha}\left(A_{i}^{\tau} \partial_{x_{i}} W^{\tau}\right)\right] .
\end{aligned}
$$

Since matrices $A_{0}^{\tau} \equiv A_{0}^{\tau}\left(n^{\tau}\right)$ and $\widetilde{A}_{i}^{\tau} \equiv A_{0}^{\tau} A_{i}^{\tau}$ are symmetric, we multiply (29) by $A_{0}^{\tau}$ and take the inner product of the resulting equations with $W_{\alpha}^{\tau}$ to get

$$
\begin{aligned}
\frac{\mathrm{d}}{\mathrm{d} t} & \left(A_{0}^{\tau} W_{\alpha}^{\tau}, W_{\alpha}^{\tau}\right)-\frac{2}{\tau^{2}}\left(A_{0}^{\tau} \partial_{x}^{\alpha} H_{2}, W_{\alpha}^{\tau}\right) \\
= & \frac{2}{\tau}\left(A_{0}^{\tau} \partial_{x}^{\alpha} H_{1}, W_{\alpha}^{\tau}\right)+\frac{2}{\tau}\left(J_{\alpha}, W_{\alpha}^{\tau}\right) \\
& +\left(\operatorname{div} A^{\tau} W_{\alpha}^{\tau}, W_{\alpha}^{\tau}\right)-2\left(A_{0}^{\tau} \partial_{x}^{\alpha} R^{\tau}, W_{\alpha}^{\tau}\right),
\end{aligned}
$$

where $(\cdot, \cdot)$ stands for the $L^{2}$ inner product of the two scalar or vector functions in $\mathbb{R}^{3}$,

$$
J_{\alpha}=-\sum_{i=1}^{3} A_{0}^{\tau}\left[\partial_{x}^{\alpha}\left(A_{i}^{\tau} \partial_{x_{i}} W^{\tau}\right)-A_{i}^{\tau} \partial_{x_{i}} W_{\alpha}^{\tau}\right]
$$

and

$$
\operatorname{div} A^{\tau}=\partial_{t} A_{0}^{\tau}+\frac{1}{\tau} \sum_{i=1}^{3} \partial_{x_{i}} \widetilde{A}_{i}^{\tau} .
$$

Let us give an estimate of each term of (30). First, a direct computation gives

$$
\begin{aligned}
-\left(A_{0}^{\tau} \partial_{x}^{\alpha} H_{2}, W_{\alpha}^{\tau}\right) & =\int_{\mathbb{R}^{3}}\left(h^{\prime}\left(n^{\tau}\right)\right)^{-1}\left|\partial_{x}^{\alpha} U^{\tau}\right|^{2} \partial_{x}^{\alpha} \mathrm{d} x \\
& \geqslant C^{-1}\left\|U_{\alpha}^{\tau}\right\|^{2} .
\end{aligned}
$$

On the other hand, since $A_{0}^{\tau}$ is a positive definite matrix, we get

$$
\left(A_{0}^{\tau} W_{\alpha}^{\tau}, W_{\alpha}^{\tau}\right) \geqslant C^{-1}\left\|W_{\alpha}^{\tau}\right\|^{2} .
$$

Next, we use the expression of $H_{1}$ to compute

$$
\begin{aligned}
& \left(A_{0}^{\tau} \partial_{x}^{\alpha} H_{1}, W_{\alpha}^{\tau}\right) \\
& =-\int_{\mathbb{R}^{3}}\left(n^{\tau}\right)^{-1} N_{\alpha}^{\tau} \partial_{x}^{\alpha}\left(U^{\tau} \cdot \nabla n_{\tau}+N^{\tau} \operatorname{div} u_{\tau}\right) \mathrm{d} x \\
& \quad-\int_{\mathbb{R}^{3}} \frac{1}{h^{\prime}\left(n^{\tau}\right)} U_{\alpha}^{\tau} \partial_{x}^{\alpha}\left[\left(U^{\tau} \cdot \nabla\right) u_{\tau}\right] \mathrm{d} x \\
& \quad-\int_{\mathbb{R}^{3}} \frac{1}{h^{\prime}\left(n^{\tau}\right)} U_{\alpha}^{\tau} \partial_{x}^{\alpha}\left[\left(h^{\prime}\left(n^{\tau}\right)-h^{\prime}\left(n_{\tau}\right)\right) \nabla n_{\tau}\right] \mathrm{d} x .
\end{aligned}
$$

Then by Lemma 1 and $u_{\tau}=O(\tau)$, we get

$$
\begin{aligned}
& \frac{2}{\tau}\left(A_{0}^{\tau} \partial_{x}^{\alpha} H_{1}, W_{\alpha}^{\tau}\right) \\
& \quad \leqslant \frac{C}{\tau}\left(\left\|N^{\tau}\right\|_{s}\left\|U^{\tau}\right\|_{s}+\tau\left\|N^{\tau}\right\|_{s}^{2}+\tau\left\|U^{\tau}\right\|_{s}^{2}\right) \\
& \quad \leqslant \frac{\epsilon}{\tau^{2}}\left\|U^{\tau}\right\|_{s}^{2}+C_{\epsilon}\left\|W^{\tau}\right\|_{s}^{2} .
\end{aligned}
$$

From here on, $\epsilon$ denotes a small constant independent of $\tau$ and $C_{\epsilon}>0$ denotes a constant depending only on $\epsilon$.

Now we consider the estimate for the term containing $J_{\alpha}$. It should be shown that a direct application of Lemma 1 to $J_{\alpha}$ does not yield the desired result. We have to develop the terms in the summation of $J_{\alpha}$ to see the appearance of terms $U^{\tau}$ or $U^{\tau}+u_{\tau}$. By the definition of $\mathrm{A}_{i}^{\tau}$, we have

$$
\begin{aligned}
\partial_{x}^{\alpha}\left(A_{i}^{\tau} \partial_{x_{i}} W^{\tau}\right)-A_{i}^{\tau} \partial_{x_{i}} W_{\alpha}^{\tau} & \left(\begin{array}{c}
\partial_{x}^{\alpha}\left(\left(u^{\tau}\right)_{i} \partial_{x_{i}} N^{\tau}\right)-\left(u^{\tau}\right)_{i} \partial_{x}^{\alpha} \partial_{x_{i}} N^{\tau} \\
\partial_{x}^{\alpha}\left(h^{\prime}\left(n^{\tau}\right) N^{\tau} e_{i}\right)-h^{\prime}\left(n^{\tau}\right) \partial_{x}^{\alpha} \partial_{x_{i}} N^{\tau} e_{i}
\end{array}\right) \\
& +\left(\begin{array}{c}
\partial_{x}^{\alpha}\left(n^{\tau} \partial_{x_{i}} U^{\tau} \cdot \mathrm{e}_{i}^{t}\right)-n^{\tau} \partial_{x}^{\alpha} \partial_{x_{i}} U^{\tau} \cdot \mathrm{e}_{i}^{t} \\
\partial_{x}^{\alpha}\left(\left(u^{\tau}\right)_{i} \partial_{x_{i}} U^{\tau}\right)-\left(u^{\tau}\right)_{i} \partial_{x}^{\alpha} \partial_{x_{i}} U^{\tau}
\end{array}\right) .
\end{aligned}
$$


Then

$$
\begin{gathered}
\left(A_{0}^{\tau}\left[\partial_{x}^{\alpha}\left(A_{i}^{\tau} \partial_{x_{i}} W^{\tau}\right)-A_{i}^{\tau} \partial_{x_{i}} W_{\alpha}^{\tau}\right], W_{\alpha}^{\tau}\right) \\
=\left(\frac{1}{n^{\tau}}\left(\partial_{x}^{\alpha}\left(u_{i}^{\tau} \partial_{x_{i}} N^{\tau}\right)-u_{i}^{\tau} \partial_{x}^{\alpha} \partial_{x_{i}} N^{\tau}\right), N_{\alpha}^{\tau}\right) \\
+\left(\frac{1}{h^{\prime}}\left(\partial_{x}^{\alpha}\left(h^{\prime} N^{\tau} e_{i}\right)-h^{\prime} \partial_{x}^{\alpha} \partial_{x_{i}} N^{\tau} e_{i}\right), U_{\alpha}^{\tau}\right) \\
+\left(\frac{1}{n^{\tau}}\left(\partial_{x}^{\alpha}\left(n^{\tau} \partial_{x_{i}} U^{\tau} \cdot \mathrm{e}_{i}^{t}\right)-n^{\tau} \partial_{x}^{\alpha} \partial_{x_{i}} U^{\tau} \cdot \mathrm{e}_{i}^{t}\right), N_{\alpha}^{\tau}\right) \\
+\left(\frac{1}{h^{\prime}}\left(\partial_{x}^{\alpha}\left(u_{i}^{\tau} \partial_{x_{i}} U^{\tau}\right)-u_{i}^{\tau} \partial_{x}^{\alpha} \partial_{x_{i}} U^{\tau}\right), U_{\alpha}^{\tau}\right) \\
=J_{i 1}+J_{i 2}+J_{i 3}+J_{i 4},
\end{gathered}
$$

where $h^{\prime}=h^{\prime}\left(n^{\tau}\right)$. Noting (18) for $u_{\tau}$ and applying Lemma 1 to each term on the right-hand side of the above equation gives

$$
\begin{aligned}
\left|J_{i 1}+J_{i 4}\right| & \leqslant C\left(\tau+\left\|U^{\tau}\right\|_{s}\right)\left\|W^{\tau}\right\|_{s}^{2} \\
& \leqslant \frac{C}{\tau}\left\|U^{\tau}\right\|_{s}^{2}+C_{\epsilon} \tau\left(\left\|W^{\tau}\right\|_{s}^{2}+\left\|W^{\tau}\right\|_{s}^{4}\right), \\
\left|J_{i 2}+J_{i 3}\right| & \leqslant C\left(1+\left\|N^{\tau}\right\|_{s}\right)\left\|N^{\tau}\right\|_{s}\left\|U^{\tau}\right\|_{s} \\
& \leqslant \frac{C}{\tau}\left\|U^{\tau}\right\|_{s}^{2}+C_{\epsilon} \tau\left(\left\|W^{\tau}\right\|_{s}^{2}+\left\|W^{\tau}\right\|_{s}^{4}\right),
\end{aligned}
$$

which implies that

$$
\frac{2}{\tau}\left(J_{\alpha}, W_{\alpha}^{\tau}\right) \leqslant \frac{\epsilon}{\tau^{2}}\left\|U^{\tau}\right\|_{s}^{2}+C_{\epsilon}\left(\left\|W^{\tau}\right\|_{s}^{2}+\left\|W^{\tau}\right\|_{s}^{4}\right) .
$$

From (20), we have obviously

$$
\begin{aligned}
& -2\left(A_{0}^{\tau} \partial_{x}^{\alpha} R^{\tau}, W_{\alpha}^{\tau}\right) \\
& \quad=-2\left(\frac{1}{n} \partial_{x}^{\alpha} R_{n}^{\tau}, N_{\alpha}^{\tau}\right)-2\left(\left(h\left(n^{\tau}\right)\right)^{-1} \partial_{x}^{\alpha} R_{u}^{\tau}, U_{\alpha}^{\tau}\right) \\
& \quad \leqslant \frac{\epsilon}{\tau^{2}}\left\|U^{\tau}\right\|_{s}^{2}+C\left\|W_{1}^{\tau}\right\|_{s}^{2}+C_{\epsilon} \tau^{2(\lambda+1)}
\end{aligned}
$$

Finally, it follows from (31) and (27) that

$$
\begin{aligned}
& \left(\operatorname{div} A^{\tau} W_{\alpha}^{\tau}, W_{\alpha}^{\tau}\right) \\
& \quad=\left(\partial_{t} A_{0}^{\tau}+\frac{1}{\tau} \sum_{i=1}^{3} \partial_{x_{i}} \widetilde{A}_{i}^{\tau} W_{\alpha}^{\tau}, W_{\alpha}^{\tau}\right) \\
& =\left(\frac{\operatorname{div} u^{\tau}}{\tau}\left(A_{0}^{\tau}-n^{\tau}\left(A_{0}^{\tau}\right)^{\prime}\right) W_{\alpha}^{\tau}, W_{\alpha}^{\tau}\right),
\end{aligned}
$$

where (1a) and (1b) have been used. Noting

$$
\begin{gathered}
\frac{\bar{n}}{2} \leqslant n^{\tau}=N^{\tau}+n_{\tau} \leqslant C_{0}, \\
u^{\tau}=U^{\tau}+u_{\tau}, \quad u_{\tau}=O(\tau),
\end{gathered}
$$

we get

$$
\left\|\operatorname{div} u^{\tau}\right\|_{\infty} \leqslant C\left\|\operatorname{div}\left(U^{\tau}+u_{\tau}\right)\right\|_{s-1} \leqslant C\left(\left\|U^{\tau}\right\|_{s}+\tau\right) .
$$

Then we deduce that

$$
\begin{aligned}
& \left(\operatorname{div} A^{\tau} W_{\alpha}^{\tau}, W_{\alpha}^{\tau}\right) \\
& \quad \leqslant C\left(1+\frac{U^{\tau}}{\tau}\right)\left(W_{\alpha}^{\tau}, W_{\alpha}^{\tau}\right) \\
& \quad \leqslant \frac{\epsilon}{\tau^{2}}\left\|U^{\tau}\right\|_{s}^{2}+C_{\epsilon}\left(\left\|W^{\tau}\right\|_{s}^{2}+\left\|W^{\tau}\right\|_{s}^{4}\right) .
\end{aligned}
$$

Thus, together with (30) and (32)-(37), we obtain, for all $|\alpha| \leqslant s$, that

$$
\begin{aligned}
& \frac{\mathrm{d}}{\mathrm{d} t}\left(A_{0}^{\tau} W_{\alpha}^{\tau}, W_{\alpha}^{\tau}\right)+\frac{k}{\tau^{2}}\left\|U_{\alpha}^{\tau}\right\|_{s}^{2} \\
& \quad \leqslant C_{\epsilon}\left(\left\|W^{\tau}\right\|_{s}^{2}+\left\|W^{\tau}\right\|_{s}^{4}\right)+C_{\epsilon}\left\|U^{\tau}\right\|_{s}^{2}+C_{\epsilon} \tau^{2(\lambda+1)} .
\end{aligned}
$$

Integrating (38) over $(0, t)$ with $t \in\left(0, T^{\tau}\right) \subset\left(0, T_{1}\right)$ and summing over all $|\alpha| \leqslant s$, taking $\epsilon>0$ sufficiently small such that the term including $C_{\epsilon}\left\|U^{\tau}\right\|_{s}^{2}$ can be controlled by the left-hand side, together with condition (19) for the initial data and noting (33), we get (28).

By the estimates in Lemma 2, for $t \in\left(0, T^{\tau} \subset\right.$ $\left.\left(0, T_{1}\right)\right)$, we have

$$
\begin{aligned}
& \left\|W^{\tau}(t)\right\|_{s}^{2}+\frac{1}{\tau^{2}} \int_{0}^{t}\left\|U^{\tau}(\xi)\right\|_{s}^{2} \mathrm{~d} \xi \\
& \quad \leqslant C \int_{0}^{t}\left(\left\|W^{\tau}(\xi)\right\|_{s}^{2}+\left\|W^{\tau}(\xi)\right\|_{s}^{4}\right) \mathrm{d} \xi+C \tau^{2(\lambda+1)} .
\end{aligned}
$$

Set

$$
\chi(t)=C \int_{0}^{t}\left(\left\|W^{\tau}(\xi)\right\|_{s}^{2}+\left\|W^{\tau}(\xi)\right\|_{s}^{4}\right) \mathrm{d} \xi+C \tau^{2(\lambda+1)} .
$$

Then it follows from (39) that

$$
\left\|W^{\tau}(t)\right\|_{s}^{2} \leqslant \chi(t), \quad \frac{1}{\tau^{2}} \int_{0}^{t}\left\|U^{\tau}(\xi)\right\|_{s}^{2} \mathrm{~d} \xi \leqslant \chi(t),
$$

and

$$
\begin{aligned}
\chi^{\prime}(t) & \leqslant C\left(\left\|W^{\tau}(t)\right\|_{s}^{2}+\left\|W^{\tau}(t)\right\|_{s}^{4}\right) \\
& \leqslant C\left(\chi(t)+\chi^{2}(t)\right)
\end{aligned}
$$

for all $t \in\left(0, T^{\tau}\right)$ with $\chi(0)=C \tau^{2(\lambda+1)}$. We apply Gronwall's lemma to (41) to get

$$
\chi(t) \leqslant C \tau^{2(\lambda+1)} \mathrm{e}^{C t} \leqslant C \tau^{2(\lambda+1)} \mathrm{e}^{C T_{1}}, \forall t \in\left[0, T^{\tau}\right] .
$$

Hence from (40) we obtain $\left\|W^{\tau}(t)\right\|_{s} \leqslant C \tau^{\lambda+1}$ and

$$
\int_{0}^{t}\left\|U^{\tau}(\xi)\right\|_{s}^{2} \mathrm{~d} \xi \leqslant C \tau^{2} \chi(t) \leqslant C \tau^{2(\lambda+2)}
$$


for all $t \in\left[0, T^{\tau}\right]$. In particular, this implies that the sequence $\left(W^{\tau}\right)_{\tau>0}$ is bounded in $L^{\infty}\left(\left[0, T^{\tau}\right], H^{s}\left(\mathbb{R}^{3}\right)\right)$, and so is $\left(n^{\tau}, u^{\tau}\right)$. By a standard argument on the time extension of smooth solutions, we obtain $T_{3}^{\tau} \geqslant T_{1}$, i.e., $T^{\tau}=T_{1}$. This completes the proof of Theorem 2 .

Acknowledgements: This work was partially supported by the Joint Funds of the National Natural Science Foundation of China (Grant No. U1204103) and the science and technology research projects of Education Department of Henan province (Grant No. 13A110731).

\section{REFERENCES}

1. Junca S, Rascle M (2002) Strong relaxation of the isothermal Euler system to the heat equation. $Z$ Angew Math Phys 53, 239-64.

2. Marcati P, Milani A (1990) The one-dimensional Darcy's law as the limit of a compressible Euler flow. $J$ Differ Equat 84, 129-47.

3. Coulombel JF, Goudon T (2007) The strong relaxation limit of the multidimensional isothermal Euler equations. Trans Am Math Soc 359, 637-48.

4. Xu J (2010) Strong relaxation limit of multidimensional isentropic Euler equations. $Z$ Angew Math Phys 6, 389-400.

5. Yang JW, Wang S, Zhao J (2011) The relaxation-time limit in the compressible Euler-Maxwell equations. Nonlin Anal 74, 7005-11.

6. Cercignani C (1988) The Boltzmann Equation and Its Applications, Springer-Verlag, New York.

7. Chen GQ, Levermore CD, Liu TP (1994) Hyperbolic conservation laws with stiff relaxation terms and entropy. Comm Pure Appl Math 47, 787-830.

8. Jang JH (2009) Vlasov-Maxwell-Boltzmann diffusive limit. Arch Ration Mech Anal 194, 531-84.

9. Kato $\mathrm{T}$ (1975) The Cauchy problem for quasi-linear symmetric hyperbolic systems. Arch Ration Mech Anal 58, 181-205.

10. Majda A (1984) Compressible Fluid Flow and Systems of Conservation Laws in Several Space Variables, Springer-Verlag, New York.

11. Klainerman S, Majda A (1981) Singular limits of quasilinear hyperbolic systems with large parameters and the incompressible limit of compressible fluids. Comm Pure Appl Math 34, 481-524. 